\title{
DOPAMINE DEFICIENCY IN THE WEAVER MUTANT MOUSE ${ }^{1}$
}

\author{
M. J. SCHMIDT, ${ }^{2}$ B. D. SAWYER, K. W. PERRY, R. W. FULLER, M. M. FOREMAN, AND B. GHETTI*
}

The Lilly Research Laboratories, Indianapolis, Indiana 46285 and ${ }^{*}$ 'The Department of Pathology, Division of Neuropathology, Indiana University School of Medicine, Indianapolis, Indiana 46223

Received August 13, 1981; Revised November 5, 1981; Accepted November 6, 1981

\begin{abstract}
The dopamine system in weaver mutant mice (B6CBA- $A^{\text {w-J }} / A$ background) was studied. Dopamine was $27 \%$ lower in the olfactory tubercle, $77 \%$ lower in the frontal cortex, and $75 \%$ lower in the striatum of 6-month-old weaver mice compared to control mice of the same age. Norepinephrine and serotonin were not lower in these brain areas. Tyrosine hydroxylase activity in the striatum was measured with a radiometric assay and was 70\% lower in weaver mice. Examination of mice from 11 to 180 days of age revealed that the dopamine system failed to develop in weaver mice. Motor activity in individual animals was assessed using circular photocell activity cages with minimal illumination. Apomorphine and pergolide, direct dopamine agonists, increased activity more in weaver mice than in normal littermates. Amphetamine, which releases endogenous stores of dopamine, was less active in mutant mice. These findings provide suggestive evidence that postsynaptic dopamine receptors in weaver mutants might have become supersensitive as a result of lower levels of dopamine in motor areas of the brain.

Anatomical evidence of dopamine system abnormalities was found in weaver mice by examination of serial sections cut from the midbrain of mutant and normal mice. The pars compacta of the substantia nigra in weaver mice appeared hypocellular when compared with the corresponding sections from controls. Fewer large neurons were seen in the affected animals.

This study illustrates that weaver mice have specific deficiencies in the dopamine system. The weaver mouse might provide a way of examining the biochemical and behavioral effects of long term dopamine deficiency and a way to examine drugs to treat dopamine-deficient states in vivo.
\end{abstract}

The weaver mutant mouse (Sidman, 1968) is characterized behaviorally by ataxia and a fine tremor. Histologically, there is an atrophy of the cerebellum which is secondary to a failure of migration and to the degeneration of the cerebellar granule cells (Rakic and Sidman, 1973). However, there is evidence that the dopamine system also is affected in the weaver mutant. Lane et al. (1977) reported that the level of dopamine in the whole brain of adult weaver mice was $50 \%$ lower than in normal littermates. The present experiments were undertaken to better define the alterations in the dopamine system in weaver mice from a morphological, biochemical, and behaviorally pharmacological standpoint and, in this respect, to establish whether the weaver mouse could serve as a model of human disorders characterized by dopamine deficiency (e.g., Parkinson's disease).

\footnotetext{
'We would like to thank Mrs. Dorothy Meyer and Ms. Constance C. Alyea for their skillful assistance. This study was supported in part by National Institutes of Health Grant PHS-1-R01NS14426 to B. G.

${ }^{2}$ To whom correspondence should be addressed.
}

\section{Materials and Methods}

Weaver mutant mice (B6CBA- $A^{\text {w-J }} / A$ background) and heterozygous littermates were obtained from the Jackson Laboratories (Bar Harbor, ME) or raised from Jackson stock in our colony. Animals were housed in 15 $\times 20 \mathrm{~cm}$ plastic cages at 23 to $24^{\circ} \mathrm{C}$ and were given water and Purina Laboratory Chow ad libitum. Mice were weaned at 25 days of age and litters were kept together until used. Control and weaver mice were evaluated simultaneously in all assays or tests. However, due to the difficulty in obtaining sizable numbers of animals of specific ages on a given date, it was not possible to include all ages simultaneously in longitudinal experiments.

A histological examination of the substantia nigra was made in weaver and control mice. Brain tissue was dissected from mice which had been decapitated. The brain stem, including the midbrain, was fixed in $10 \%$ formalin, while the remainder was used for neurochemical determinations in some cases. The brain stems from six weaver mice and six control mice were fixed in formalin, dehy- 
drated in graded alcohols, and embedded in paraffin. Serial sections (130 to 150 ) were cut ( $7 \mu \mathrm{m}$ thick) from the midbrain and upper pons of each mouse. Sections were stained alternatively with gallocyanin for Nissl substance and with hematoxylin/eosin. These series of sections were examined without prior knowledge of the neurochemical results. Pathological findings then were verified by an outside neuropathologist completely naive to the project.

Monoamine levels in the olfactory tubercle, frontal cortex, and striatum were determined using high pressure liquid chromatography and electrochemical detection (Fuller and Perry, 1977, 1981). Concentrations of dopamine, norepinephrine, and serotonin were measured in the olfactory tubercle, frontal cortex, corpus striatum, cerebellum, and the brain stem. Data are expressed in nanomoles of neurotransmitter per gm of tissue. The amine metabolites, homovanillic acid and 5-hydroxyindoleacetic acid (5-HIAA), also were determined in these brain areas (Perry and Fuller, 1979).

Tyrosine hydroxylase was measured in the corpus striatum using a radiometric assay (Lerner et al., 1978). Enzyme activity was assayed at saturating levels of cofactor. Protein concentrations in the striatal extracts were measured according to the procedures of Lowry et al. (1951).

Motor activity was determined in the mice by placing them individually in circular activity cages $25 \mathrm{~cm}$ in diameter. Each cage was equipped with a photocell connected to a counter. Mice were allowed to adapt to the new environment, which was minimally illuminated, for $60 \mathrm{~min}$, and then, each was administered apomorphine $\mathrm{HCl}(5 \mathrm{mg} / \mathrm{kg}$, i.p.). Mice were returned to the cages and the number of photocell breaks was recorded for three successive 30-min periods. After a 10-day "washout," animals were administered pergolide mesylate, a direct acting dopamine agonist in the ergoline class (Fuller et al., 1979), at $1 \mathrm{mg} / \mathrm{kg}$ using the above paradigm. Ten days after the pergolide experiment, animals were administered $2.5 \mathrm{mg} / \mathrm{kg}$ of amphetamine sulfate (intraperitoneally), and activity was recorded for $120 \mathrm{~min}$. Animals were sacrificed by decapitation, and neurochemical determinations of monoamines were made 2 weeks after the amphetamine experiment. The dopamine concentrations in weaver mice or control mice not administered amphetamine or dopamine agonists were the same as animals that had been given drugs. Therefore, data from "drug-naive" animals and animals previously exposed to the drugs were combined in computing the neurochemical results.

\section{Results}

Histology. The pars compacta of the substantia nigra in 6-month-old weaver mice appeared hypocellular when compared with the corresponding region in control littermates (Fig. 1). In particular, fewer large neurons were seen in the affected animals. The neuronal population of the nucleus was not counted, and, although the histological differences were remarkable, the present data need confirmation by quantitative analysis.

Neurochemistry. There was a reduction in the concen- tration of dopamine in a number of brain areas of 6 month-old weaver mutant mice (Table I). The level of dopamine was $28 \%$ lower in the olfactory tubercle, $77 \%$ lower in the frontal cortex, and $75 \%$ lower in the corpus striatum. Dopamine was not decreased in the brain stem and was not present to any degree in the cerebellum. A major metabolite of dopamine, homovanillic acid, was also lower in those areas where dopamine was reduced (Table I).

'The concentration of norepinephrine was not different in control and weaver animals in any of the five brain areas. Amounts of serotonin were also similar in the control and mutant animals with the exception of the cerebellum. Serotonin levels in the cerebellum of weaver mice were $348 \%$ higher than in age-matched littermate controls (Table I). The major metabolite of serotonin, 5 HIAA, showed a similar pattern (i.e., an increase only in the carebellum).

Dopamine was measured in the corpus striatum of control and weaver mice 11 to 180 days of age (Fig. 2). Dopamine concentrations were significantly lower at the earliest time point. While dopamine increased from 20 to approximately $60 \mathrm{nmol} / \mathrm{gm}$ of tissue in the normal mice during the course of development, the maximum concentration in weaver mice was $25 \mathrm{nmol} / \mathrm{gm}$ of striatum. These amounts of dopamine decreased progressively with age in the weaver mice such that dopamine concentrations were $75 \%$ lower in 180- compared to 21 -day-old weaver mice (Fig. 2).

Tyrosine hydroxylase activity was determined in striatal tissue from control and weaver mice at 16 and also at 135 days of age (Fig. 3). Tyrosine hydroxylase activity was $43 \%$ lower in weaver mice at 16 days of age and $77 \%$ lower at 135 days of age. During this time period, tyrosine hydroxylase activity did not change in normal animals; however, there was a significant reduction in the striatum of weaver mice during this same period.

Behavioral pharmacology. Weaver mutant mice were hyper-reactive when handled and were not able to maintain their balance when placed on elevated metal discs of progressively decreasing size (M. J. Schmidt, unpublished observations). Weaver mice were also hyperactive when placed in the locomotor activity chamber (Fig. 4, $A$ to $C)$. The locomotor stimulation by apomorphine $(5 \mathrm{mg} /$ $\mathrm{kg}$ ) or pergolide $(1 \mathrm{mg} / \mathrm{kg})$, direct acting dopamine agonists (Fuller et al., 1979), was significantly greater and of longer duration in weaver mice than in control mice (Fig. $4, A$ and $B$ ). However, the stimulation by amphetamine $(2.5 \mathrm{mg} / \mathrm{kg})$, an indirect dopamine stimulant, was of lower magnitude and shorter duration in weaver mutants (Fig. $4 C)$.

\section{Discussion}

We have shown that, in the weaver mutant mouse, there is a marked reduction in the concentration of dopamine in dopamine-rich areas of the brain. Differences in weaver and normal littermates were observed as early as 11 days of age. Tyrosine hydroxylase activity was also substantially lower in the corpus striatum of weaver mice. A reduction in the number of nerve cell 

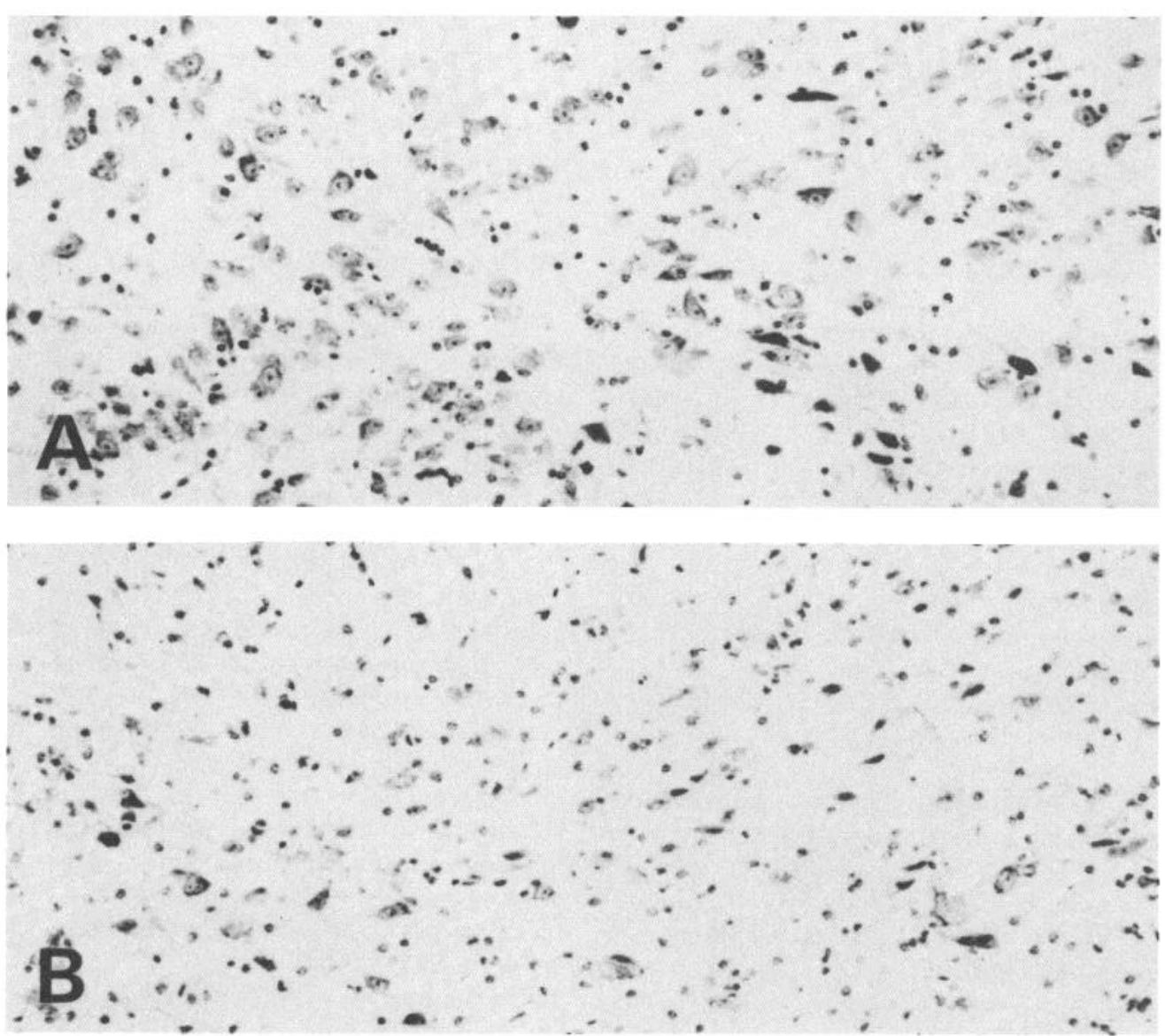

Figure 1. Substantia nigra (pars compacta) from a 6-month-old unaffected mouse $(A)$ and from a littermate weaver mutant $(B)$. Note that nerve cells in the control mouse are more numerous and larger than in the mutant.

TABLE I

Neurotransmitter and metabolite concentrations in brain areas of control and weaver mice Data represent the mean \pm SEM of six animals per group.

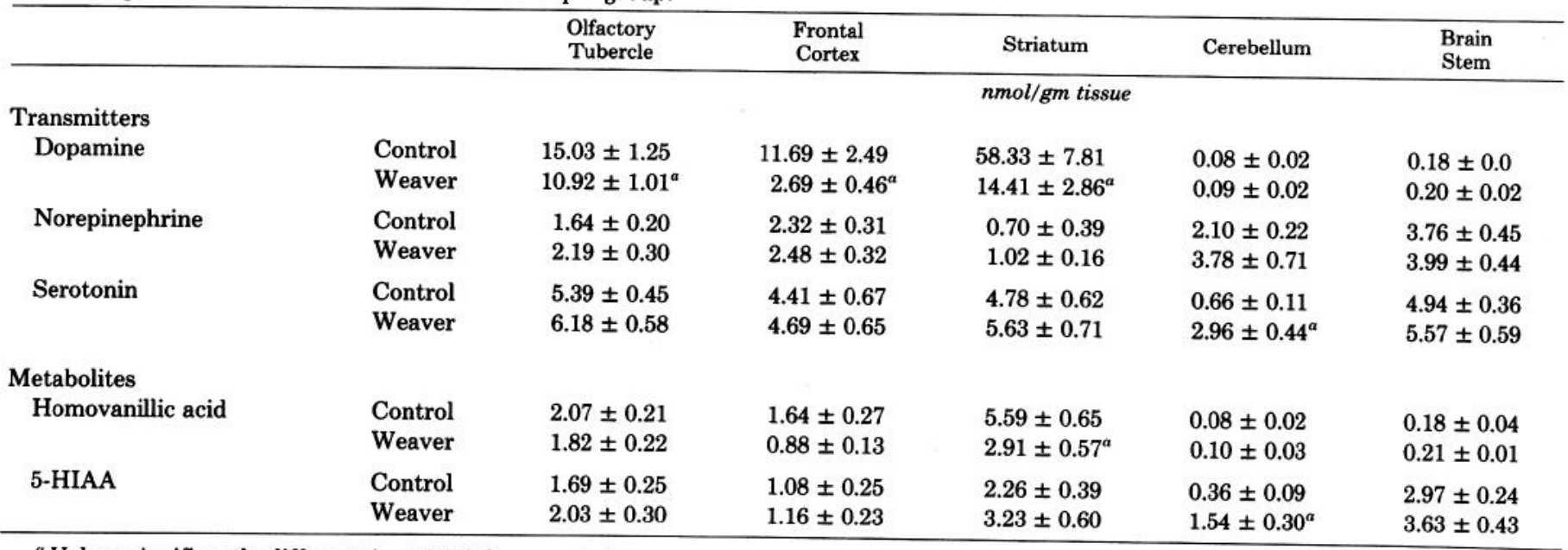

${ }^{a}$ Values significantly different $(p<0.05)$ from controls.

perikarya was detected in the substantia nigra of the weaver.

The abnormalities in the weaver mouse monoamine system appear quite specific. Dopamine concentrations and metabolites were reduced in dopamine-rich areas of the brain, but norepinephrine and serotonin were not affected (Table I). Examination of the substantia nigra revealed that, although there were fewer neurons (Fig: 1, 


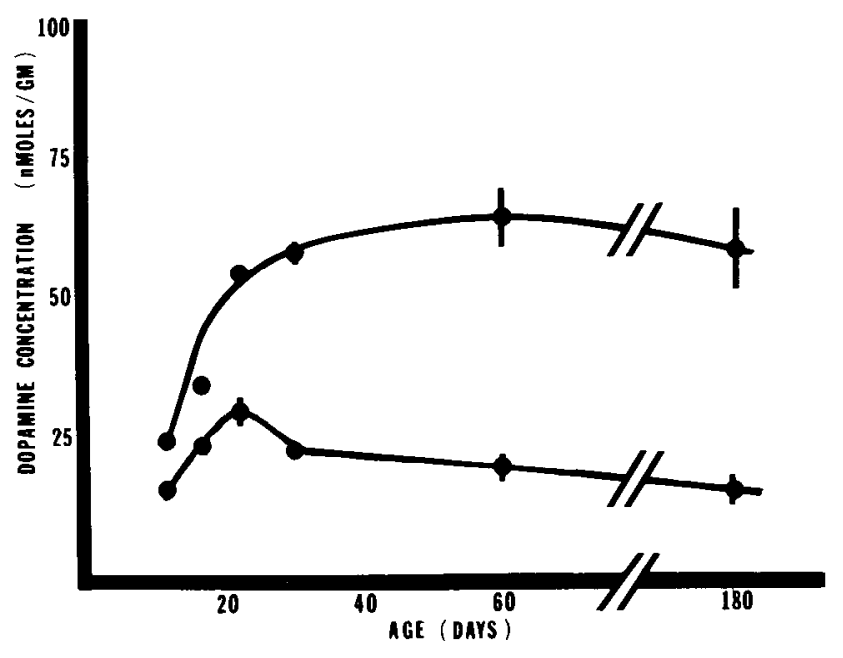

Figure 2. Striatal dopamine concentrations in control and weaver mice. The points and vertical lines represent the mean \pm SEM of six to eight animals per age group. Ages without vertical lines indicate that the standard errors are encompassed by their respective points. Upper curve, control; lower curve, weaver mutant mice.

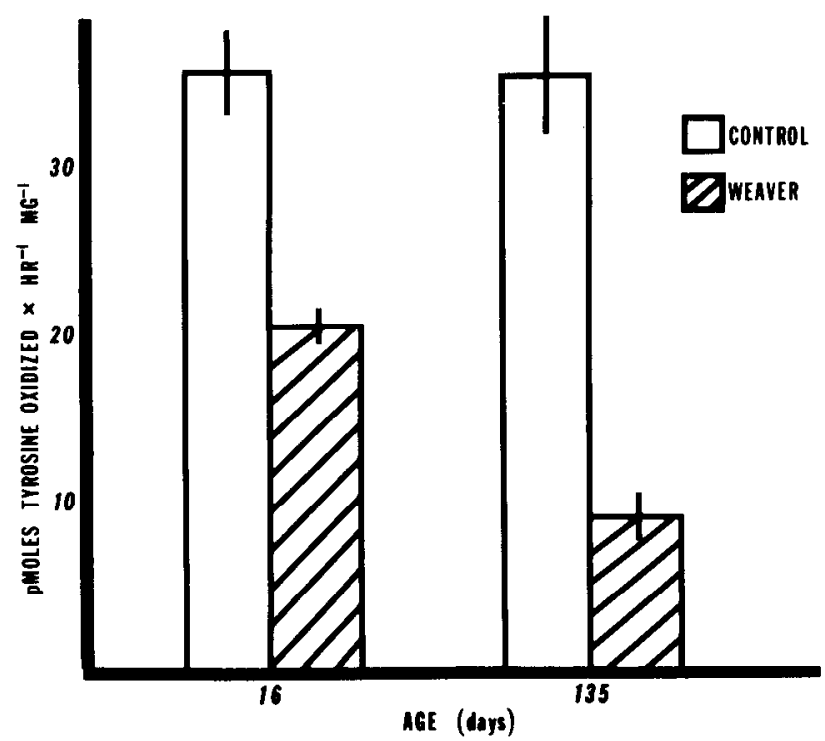

Figure 3. Comparison of striatal tyrosine hydroxylase activity between weaver mutant mice and heterozygous littermates at 16 and 135 days of age. Corpus striata were homogenized in 20 $\mathrm{vol}(\mathrm{w} / \mathrm{v})$ of distilled water with glass-glass homogenizers. The homogenates were centrifuged for $20 \mathrm{~min}$ at $1500 \times \mathrm{g}$ at $4^{\circ} \mathrm{C}$. The supernatants were assayed for tyrosine hydroxylase activity according to the method of Lerner et al. (1978). Incubations were performed at $37^{\circ} \mathrm{C}$ for $15 \mathrm{~min}$. The results are expressed as picomoles of tyrosine oxidized per hr per mg of protein.

$A$ and $B$ ), the entire area was not devoid of cells, suggesting that perhaps only the dopaminergic neurons were affected in the brain stem of the weaver mouse. The positive correlation between the loss of dopamine in the striatum and the hypocellularity of the substantia nigra in the weaver mutant is in line with reductions in dopamine in the striatum produced experimentally by chemical lesions of the substantia nigra (Ungerstedt, 1971). To our knowledge, the weaver mutant mouse represents the only natural and noninvasive model of dopamine deficiency in the brain. In this respect, it offers advantages over the other means by which dopamine-deficient states are created (e.g., electrolytic lesions or chemical lesions with 6 -hydroxydopamine). In the latter cases, the lesions are not subtle or discrete and other transmitter systems are affected in many instances. The specificity of the amine loss in the weaver now has been well documented.

Enhanced motor activity (Zigmond and Stricker, 1980) and stereotyped behavior (Ungerstedt, 1971) in response to dopaminergic drugs occur following lesions of the substantia nigra with 6-hydroxydopamine. There are indications that changes in the corpus striatum might have occurred in weaver mice as a result of the dopamine deficiency. For example, the response of the region to direct acting dopamine agonists was heightened markedly in weaver animals (Fig. $4, A$ and $B$ ), indicating supersensitive dopamine receptors. We are presently measuring dopamine receptor binding sites in the corpus striatum as a biochemical index of such supersensitivity.

There are a number of analogous changes in the dopamine system in the weaver mouse and in dopaminedeficient states in humans, such as Parkinson's disease and related disorders (Schmidt, 1981). Dopamine concentrations are lower in the striatum, there is neuronal loss in the substantia nigra, tyrosine hydroxylase activity is reduced, and Parkinson patients are initially highly responsive to dopamine agonists. However, the weaver mutant differs from the Parkinson patients in several respects. The weaver mutant has cerebellar degeneration, whereas this is not present in parkinsonism. Also, in the nerve cell perikarya of the weaver, we have not recognized cytological lesions comparable to those observed in Parkinson's disease (e.g., Lewy bodies). Furthermore, the chronology of the dopamine deficiency differs in weaver mice and Parkinson patients. The onset of Parkinson's disease occurs in mid-life at the earliest, with the greatest incidence during late middle age. In contrast, the nigrostriatal dopamine system in the weaver mouse fails to develop normally as indicated by a lower level of tyrosine hydroxylase activity (Fig. 3), lower levels of dopamine (Table I), and a plateauing of the dopamine developmental curve well below normal levels (Fig. 2). It is possible that, in the weaver, cell loss occurs very early in life, perhaps even prenatally. The fact that tyrosine hydroxylase activity declines between 16 and 180 days of age (Fig. 3) does suggest a continuing degeneration of the system, a possibility to be evaluated further by anatomical examination of the substantia nigra in weaver mice at early ages.

Although the weaver mouse does not seem to be an appropriate model to study the causes of nigrostriatal neuronal degeneration in Parkinson's disease, it does represent a noninvasive model of dopamine deficiency. New models are needed to study the causes and treatment of Parkinson's disease (Breese et al., 1978; Silbergeld and Calne, 1981) and to evaluate the therapeutic potentials and limitations in the pharmacotherapy of dopamine-deficient states. The weaver mouse might provide a way of examining the biochemical and behavioral effects of long term dopamine deficiency and a way to examine drugs to treat dopamine-deficient states in vivo. 

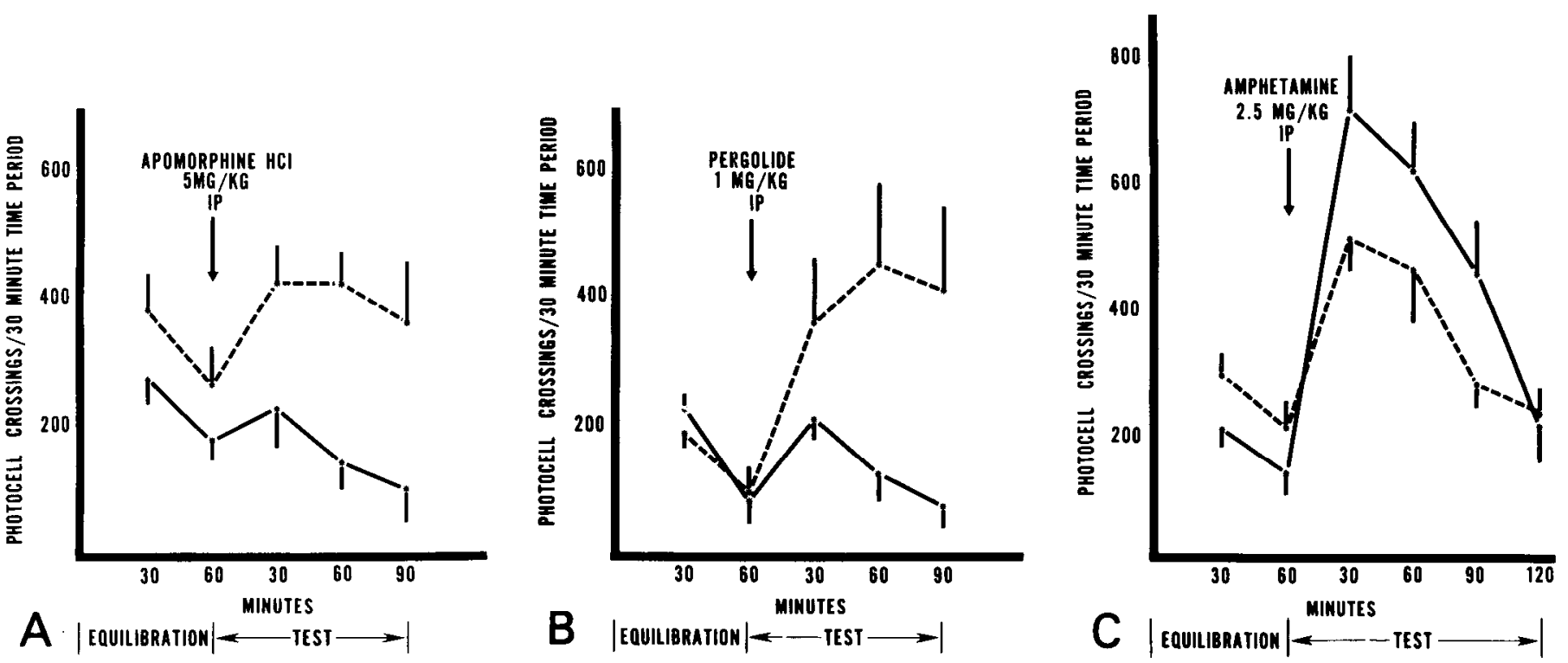

Figure 4. Mice were placed individually in circular motor activity cages and allowed to equilibrate for 60 min. Apomorphine $(A)$, pergolide $(B)$, or amphetamine $(C)$ then was administered (intraperitoneally) and activity was monitored for 90 to 120 min. Activity is expressed as the mean photocell breaks per $30 \mathrm{~min} \pm \mathrm{SEM}$ of six to eight mice per group. Control data are depicted by the solid line and weaver mice data are shown by the dashed line.

\section{References}

Breese, G. R., R. A. Mueller, R. B. Mailman, G. D. Frye, and R. A. Vogel (1978) An alternative to animal models of central nervous system disorders: Study of drug mechanisms and disease systems in animals. Prog. Neuropsychopharmacol. 2: 313-325.

Fuller, R. W., and K. W. Perry (1977) Lowering of epinephrine concentration in rat brain by 2,3 -dichloro- $\alpha$-methylbenzylamine, an inhibitor of norepinephrine $N$-methyltransferase. Biochem. Pharmacol. 26: 2087-2090.

Fuller, R. W., and K. W. Perry (1981) Antagonism by (-)methadone of the depletion of 5-hydroxytryptamine in rat brain by $p$-chloroamphetamine. J. Pharm. Pharmacol. 33: 48-49.

Fuller, R. W., J. A. Clemens, E. C. Kornfeld, H. D. Snoddy, E.

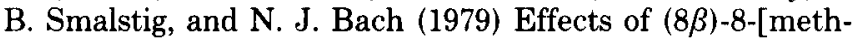
ylthio)methyl]-6-propylergoline on dopaminergic function and brain dopamine turnover in rats. Life Sci. 2: 375-382.

Lane, J. D., N. S. Nadi, W. J. McBride, M. H. Aprison, and K. Kusano (1977) Contents of serotonin, norepinephrine and dopamine in the cerebrum of the "staggerer," "weaver" and "nervous" neurologically mutant mice. J. Neurochem. 29: 349-350.

Lerner, P., P. Nose, M. Ames, and W. Lovenberg (1978) Modification of the tyrosine hydroxylase assay in the presence of ascorbic acid. Neurochem. Res. 3: 641-651.
Lowry, O. H., N. J. Rosebrough, A. L. Farr, and R. J. Randall (1951) Protein measurement with the Folin phenol reagent. J. Biol. Chem. 193: 265-275.

Perry, K. W., and R. W. Fuller (1979) Analysis of biogenic amine metabolites in rat brain by HPLC with electrochemical detection. Soc. Neurosci. Abstr. 5: 348.

Rakic, P., and R. L. Sidman (1973) Sequence of developmental abnormalities leading to granule cell deficit in cerebellar cortex of weaver mutant mice. J. Comp. Neurol. 152: 103-132.

Schmidt, M. J. (1981) The pharmacotherapy of Parkinson's disease. In Neuropharmacology of Central Nervous System and Behavioral Disorders, pp. 149-171, Academic Press, New York.

Sidman, R. L. (1968) Development of interneuronal connections in brains of mutant mice. In Physiological and Biochemical Aspects of Nervous Integration, F. D. Carlson, ed., pp. 163-193, Prentice-Hall, Englewood Cliffs, NJ.

Silbergeld, E. K., and D. B. Calne (1981) Animal models of parkinsonism. Pharmacol. Ther. 12: 159-166.

Ungerstedt, U. (1971) Postsynaptic supersensitivity after 6-hydroxydopamine-induced degeneration of the nigro-striatal dopamine system. Acta Physiol. Scand. Suppl. 367: 69-93.

Zigmond, M. J., and E. M. Stricker (1980) Supersensitivity after intraventricular 6-hydroxydopamine: Relation to dopamine depletion. Experientia 36: 436-438. 